\title{
Combined analysis of Rac1, IQGAP1, Tiam1 and E-cadherin expression in gastric cancer
}

\begin{abstract}
Axel Walch ${ }^{1, *}$, Stefan Seidl ${ }^{2, *}$, Christine Hermannstädter ${ }^{2}$, Sandra Rauser ${ }^{1}$, Joëlle Deplazes ${ }^{2}$, Rupert Langer ${ }^{2}$, Claus Hann von Weyhern ${ }^{2}$, Mario Sarbia ${ }^{3}$, Raymonde Busch $^{4}$, Marcus Feith ${ }^{5}$, Sonja Gillen ${ }^{5}$, Heinz Höfler ${ }^{1,2}$ and Birgit Luber ${ }^{2}$

${ }^{1}$ Helmholtz Zentrum München, Deutsches Forschungszentrum für Gesundheit und Umwelt (GmbH), Institut für Pathologie, Neuherberg, Germany; ${ }^{2}$ Technische Universität München, Klinikum rechts der Isar, Institut für Allgemeine Pathologie und Pathologische Anatomie, München, Germany; ${ }^{3}$ Krankenhaus Lichtenberg, Institut für Pathologie, Berlin, Germany; ${ }^{4}$ Technische Universität München, Klinikum rechts der Isar, Institut für Medizinische Statistik und Epidemiologie, München, Germany and ${ }^{5}$ Technische Universität München, Klinikum rechts der Isar, Chirurgische Klinik und Poliklinik, München, Germany
\end{abstract}

\begin{abstract}
Rho GTPases are a family of major regulators of E-cadherin-mediated cell adhesion that are implicated in the carcinogenic process by deregulated expression of the family members itself or of upstream modulators or downstream effectors. Combined investigation of the Rho GTPase Rac1, the effector protein IQGAP1 and the activator Tiam1 in relation to expression or mutation of E-cadherin in gastric adenocarcinomas has not been reported. The aim of the study was to determine the expression and prognostic significance of Rac1, IQGAP1, Tiam1 and E-cadherin in gastric adenocarcinomas. Gastric carcinomas of 76 patients were investigated immunohistochemically in a tissue microarray study for expression of Rac1, IQGAP1, Tiam1 and E-cadherin. Correlations with clinical and follow-up data were examined. Moderate or strong reactivity for Rac1 was observed in $46 \%$ and for Tiam 1 in $56 \%$ of tumors. Expression of IQGAP1 was present in $59 \%$ and of E-cadherin in $87 \%$ of tumors. While Rac1 and E-cadherin expression were not related to prognosis, a trend was observed between a lack of IQGAP1 expression (log-rank 0.088) as well as presence of Tiam1 (log-rank 0.097) and favorable prognosis in Kaplan-Meier survival analysis. Expression of Rac1 was positively linked to IQGAP1 expression $(P=0.007, r=0.343)$ and tended to be inversely associated with expression of $E$-cadherin $(P=0.055$, $r=-0.245)$. In conclusion, we observed deregulated expression of Rac1, IQGAP1, Tiam1 and E-cadherin in gastric cancer. We present evidence that either upregulation (for Rac1 and IQGAP1) or downregulation (for Tiam1 and E-cadherin) occurs. Rac1 and E-cadherin expression were not related to prognosis, while trends pointing to favorable prognosis of patients with Tiam1 expression and a lack of IQGAP1 expression were observed. These results indicate that the investigated regulators of E-cadherin-mediated cell adhesion play a role in gastric carcinogenesis.

Modern Pathology (2008) 21, 544-552; doi:10.1038/modpathol.2008.3; published online 1 February 2008
\end{abstract}

Keywords: E-cadherin; gastric carcinoma; IQGAP1; Rac1; Rho GTPase; Tiam1

Rho GTPases regulate key signalling processes of cells including E-cadherin-mediated cell adhesion, cell polarity, apoptosis and proliferation. ${ }^{1,2}$ Recently, Rho GTPases have been implicated in different aspects of the tumor development: dedifferentiation, upregulation of uncontrolled proliferation, angiogenesis, invasion and metastasis. ${ }^{3}$

Correspondence: Dr B Luber, PhD, Institut für Allgemeine Pathologie und Pathologische Anatomie, Technische Universität München, Trogerstrasse 18, 81675 München, Germany.

E-mail: luber@lrz.tu-muenchen.de

*A Walch and S Seidl contributed equally and share the first authorship.

Received 18 June 2007; revised 11 December 2007; accepted 31 December 2007; published online 1 February 2008
Rho GTPases are regulated by a mechanism that depends on their ability to cycle between an active GTP-bound state and an inactive GDP-bound state. This regulatory cycle is under the control of three distinct families of proteins, guanine exchange factors, GTPase-activating proteins and guanine nucleotide dissociation inhibitors. Upon GTP-loading, Rho GTPases interact with a large number of cellular proteins. These downstream effectors ultimately transmit the signal within the cell.

Rho GTPases are implicated in the carcinogenic process by deregulated expression of the family members itself or of upstream modulators or downstream effectors. ${ }^{3}$ Overexpression of the Rho GTPases is likely to result in an increase in the 
turnover of GTP-loading, leading to overactivation of subsequent downstream signalling. So far, activating point mutations in the GTP-binding domain of Rho GTPases have not been detected, which is a major difference between Ras and Rho GTPases.

Rho GTPases are essential for the formation of adherens junctions. The key adhesion molecule in adherens junctions is E-cadherin. Rac1 regulates in concert with Rho and Cdc42 the establishment and maintenance of adherens junctions in epithelial cells. ${ }^{4}$ The GEF Tiam1 has been identified as a specific activator of Rac1. ${ }^{5}$ Tiam-1-mediated activation of Rac1 stimulates cadherin-mediated cell-cell adhesion. ${ }^{6}$ In contrast, IQGAP1 is an effector of Rac1 that accumulates at sites of cell-cell contact and plays the role of a negative regulator of cell adhesion. $^{7-9}$

Gastric carcinoma is one of the leading malignancies worldwide that is frequently associated with mutations or downregulation of the cell adhesion protein and tumor suppressor E-cadherin. ${ }^{10}$ Somatic E-cadherin mutations that impair the adhesive function of the protein have been described in diffuse type gastric carcinomas. ${ }^{11-16}$ The predominant type of mutations in gastric cancer were splice-site mutations and in-frame deletions and most of the mutations were located in exons 8 or $9 .^{16,17}$

Although a number of in vitro investigations have established the significance of Rac1, IQGAP1 and Tiam1 as regulators of E-cadherin-mediated cell adhesion, little is known about the relevance of these markers in gastric cancer. Therefore, the aim of the study was to determine the expression and prognostic significance of Rac1, IQGAP1 and Tiam1 in gastric cancer. Further, we investigated whether the expression of these markers was correlated with each other or with the expression level of E-cadherin.

\section{Materials and methods}

\section{Patient Selection}

Patients $(n=76)$ with gastric adenocarcinoma according to the World Health Organization classification were included in the tissue microarray. ${ }^{18}$ All patients underwent primary surgical resection without chemo- or radiotherapy at the Department of Surgery, Klinikum rechts der Isar, Technische Universität München, between 1990 and 2000. TNM status was determined according to the current International Union Against Cancer classification. ${ }^{19}$ Data were acquired with approval from the ethics committee of the Technische Universität München. A minimum follow-up of at least 10 years was available for 60 patients. All 76 tumors were screened for the presence of the mutant E-cadherin variant with deletion of exon 9 with a mutationspecific E-cadherin antibody and one tumor with del 9 mutation was identified. In addition, a second gastric adenocarcinoma with del 9 mutation was detected by routine examination and included into the study.

\section{Construction of Tissue Microarrays}

Paraffin wax blocks of 76 patients were obtained from the archives of the Institute of Pathology of the Technische Universität München. Representative, non-necrotic tumor areas were selected on hematoxylin-eosin-stained slides from these blocks. Three tissue cylinders per tumor with a diameter of $0.6 \mathrm{~mm}$ covering these areas were obtained from the paraffin blocks by core-needle biopsies using a manual arrayer (Beecher Instruments, Sun Prairie, WI, USA) and positioned in a recipient paraffin wax array block.

\section{Antibodies}

The following antibodies were used for immunohistochemistry: monoclonal anti-human Rac1 antibody (BD Transduction laboratories, clone 102), monoclonal anti-human IQGAP1 antibody (BD Transduction laboratories, clone 24), rabbit antihuman Tiam1 antibody (Calbiochem, Merck KGaA, Darmstadt, Germany No. ST1070) and monoclonal anti-E-cadherin antibody HECD-1 (Alexis Deutschland, Grünberg, Germany), mutation-specific antibody recognizing the mutant E-cadherin variant lacking exon $9 .^{20}$

\section{Immunohistochemical Analysis}

Manual staining protocols were used with the following dilutions: Rac1 1:7500, IQGAP1 1:2500, Tiam1 1:500 and E-cadherin 1:500. Antigen retrieval was performed using citrate buffer using a microwave (for Rac1 and IQGAP1 staining) or a pressure cooker (for Tiam1 and E-cadherin staining). A peroxidase block $\left(3 \% \mathrm{H}_{2} \mathrm{O}_{2}\right.$ for $15 \mathrm{~min}$ at room temperature), an avidin biotin block (Vectastain, $2 \times 15 \mathrm{~min}$ at room temperature) and blocking with $5 \%$ anti-goat serum in Dako dilution solution $(2 \mathrm{~h}$, room temperature) was performed. Staining was carried out with LSAB-DAB from Dako Diagnostika $\mathrm{GmbH}$ (Hamburg, Germany). As positive controls, we used breast carcinoma (for Rac1), gastric mucosa (for IQGAP1 and E-cadherin) and colon carcinoma as well as mouse skin (for Tiam1). In addition, formalin-fixed paraffin-embedded cell pellets from MDA-MB-435S cells transfected with wild-type E-cadherin were used as positive controls because these cells express all the investigated markers. Immunohistochemical analysis of mutant del $9 \mathrm{E}$ cadherin was carried out as described previously. ${ }^{21}$ The immunohistochemical analyses were carried out with the 76 tumor samples on the tissue 
microarray and for the two cases with del 9 mutation the whole paraffin blocks were used.

\section{Reactivity Score and Interpretation of the Immunohistochemical Staining}

Rac1 staining was cytoplasmic, IQGAP1 staining membranous and cytoplasmic and Tiam1 staining membranous in tumor cells. For Rac1, IQGAP1 and Tiam1, tumors were considered as negative, when no staining or staining in $<10 \%$ neoplastic cells was observed. Weak staining in $>10 \%$ neoplastic cells was considered as $1+$ positive, moderate staining in $>10 \%$ neoplastic cells as $2+$ positive and strong staining in $>10 \%$ neoplastic cells as $3+$ positive.

For the analysis of E-cadherin staining, tumors were considered as negative, when no staining was detectable. Membranous staining in $<10 \%$ neoplastic cells was evaluated as $1+$ positive, in $>10$ and $<50 \%$ of neoplastic cells as $2+$ positive and in $>50 \%$ of neoplastic cells as $3+$ positive. Tumors were considered as del 9 positive when membranous staining was detected in tumor cells, without quantifying the percentage of positive tumor cells.

Staining of tissue microarrays for Rac1, IQGAP1, Tiam1 and E-cadherin was interpreted only when identical results were obtained for all three-tumor spots (Table 2). Rac1 staining was interpretable in 67 cases ( $88 \%$ of all cases), IQGAP1 staining in 63 cases (89\% of all cases), Tiam1 staining in 71 cases (93\% of all cases) and E-cadherin staining in 69 cases (91\% of all cases).

\section{Statistical Analysis}

Evaluation was performed by two investigators (AW and BL) who were unaware of clinical features and survival. Statistical analyses were performed using Fisher's exact, Kruskal-Wallis or $\chi^{2}$ tests when appropriate. Kaplan-Meier survival time analysis was used to correlate Rac1, IQGAP1, Tiam1 and E-cadherin reactivity, $\mathrm{pT}$ and $\mathrm{pN}$ with clinical evolution. Cox regression analysis was performed correlating the investigated markers with prognosis. Correlation analysis between the investigated markers was performed using the Spearman's rho test. A two-sided $P$-value less than 0.05 was considered to be statistically significant.

\section{Results}

\section{Immunohistochemical Expression of Rac1, IQGAP1,} Tiam1 and E-cadherin

In normal gastric mucosa, negative or weakly positive cytoplasmic Rac1 staining in surface epithelium was observed. Lymphocytes were positive for Rac1 and this staining served as internal control. Further, membrane staining of surface epithelium was detected for IQGAP1. Moderate to strong membranous Tiam1 staining and strong membranous E-cadherin staining of epithelial cells was detected in non-cancerous gastric mucosa.

The clinical and pathological features of the gastric cancer patients are shown in Table 1. Rac1 reactivity was absent in two cases ( $3 \%$, score 0$)$, weak in 34 cases $(51 \%$, score $1+$ ), moderate in 26 cases $(39 \%$, score $2+)$ and strong in five cases $(7 \%$, score $3+$ ). Rac1 expression was detected in the cytoplasm of the tumor cells as well as in lymphocytes. Examples for adenocarcinomas displaying cytoplasmic staining of Rac1 are shown in Figure 1a-d.

IQGAP1 reactivity was absent in 26 cases $(41 \%$, score 0 ), weak in 29 cases $(46 \%$, score $1+)$, moderate in six cases $(10 \%$, score $2+)$ and strong in two cases ( $3 \%$, score $3+$, Table 2$)$. Immunostaining of IQGAP1 was observed at the cellular membrane and in the cytoplasm of tumor cells (Figure 1e-h). Lymphocytes and granulocytes showed membranous IQGAP1 expression.

Tiam 1 reactivity was absent in five cases $(7 \%$, score 0 ), weak in 26 cases $(37 \%$, score $1+)$, moderate in 32 cases ( $45 \%$, score $2+$ ) and strong

Table 1 Clinicopathologic features of 76 patients with gastric cancer

\begin{tabular}{|c|c|c|}
\hline $\begin{array}{l}\text { Age } \\
\quad \text { Mean } \\
\text { Median } \\
\text { s.d. } \\
\text { Range }\end{array}$ & $\begin{array}{c}\text { Years } \\
65.0 \\
69.0 \\
12.5 \\
30-86\end{array}$ & \\
\hline & $n$ & $\%$ \\
\hline \multicolumn{3}{|l|}{ Gender } \\
\hline Female & 20 & 26 \\
\hline Male & 56 & 74 \\
\hline \multicolumn{3}{|l|}{ Histotype (Laurén) } \\
\hline Intestinal & 53 & 70 \\
\hline Diffuse and mixed & 23 & 30 \\
\hline \multicolumn{3}{|l|}{ Tumor stage } \\
\hline pT1 & 12 & 16 \\
\hline pT2a and pT2b & 42 & 58 \\
\hline pT3 & 17 & 23 \\
\hline pT4 & 2 & 3 \\
\hline \multicolumn{3}{|c|}{ Perigastric lymph node status } \\
\hline No & 24 & 33 \\
\hline N1 & 28 & 39 \\
\hline N2 & 20 & 28 \\
\hline \multicolumn{3}{|l|}{ Grading } \\
\hline 1 & 2 & 3 \\
\hline 2 & 26 & 36 \\
\hline 3 & 44 & 61 \\
\hline \multicolumn{3}{|l|}{ Residual disease } \\
\hline R0 & 72 & 99 \\
\hline R1 & 1 & 1 \\
\hline
\end{tabular}



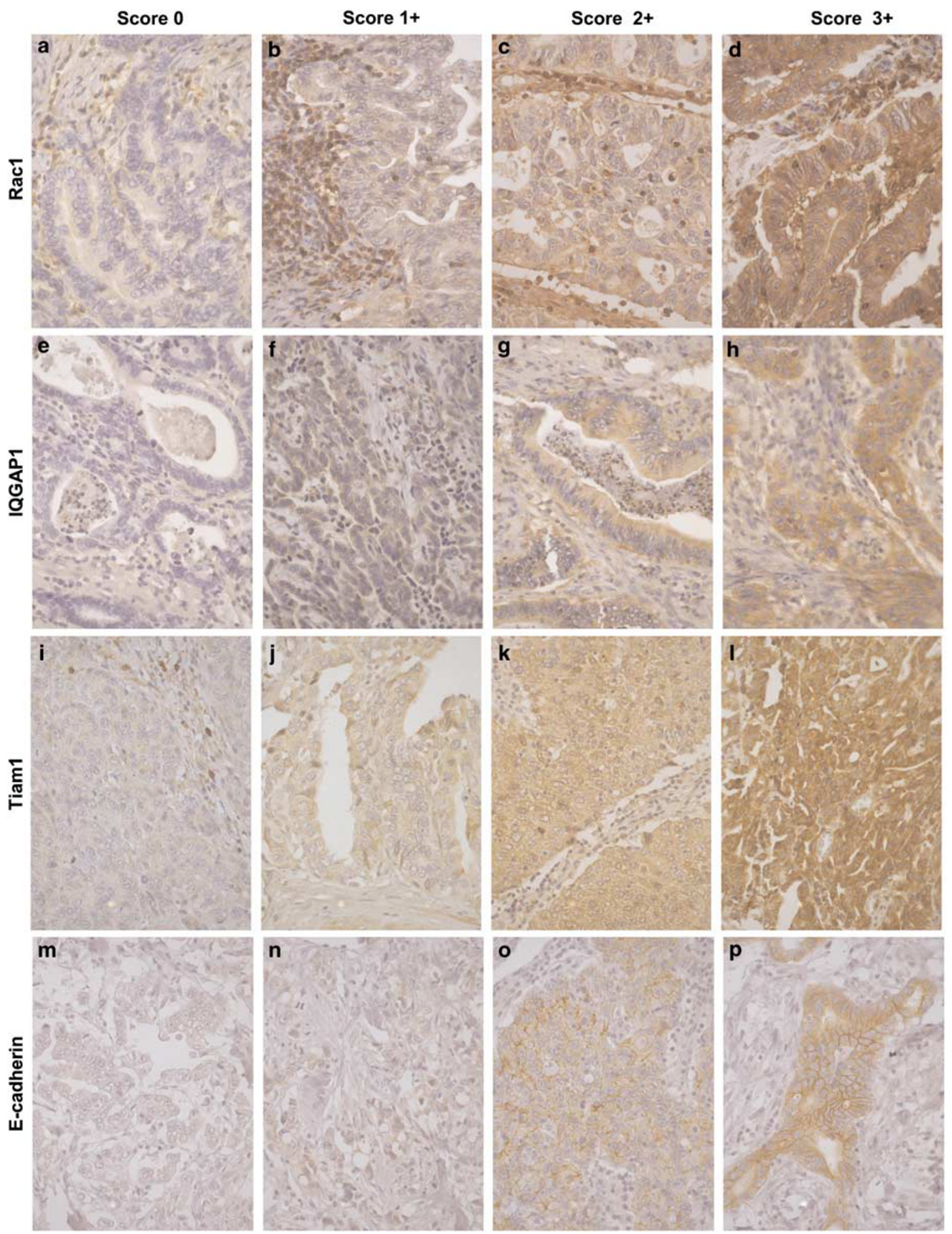

Figure 1 Immunohistochemical staining for Rac1, IQGAP1, Tiam1 and E-cadherin in gastric adenocarcinoma. Adenocarcinoma negative for Rac1 (a) or displaying weak cytoplasmic staining of Rac1 with an intensity of $1+$ (b), an example Rac1 for expression with a moderate intensity of $2+(\mathbf{c})$ or strong Rac1 staining with an intensity of $3+(\mathbf{d})$ are shown. Gastric cancer samples negative for IQGAP1 (e) or showing weak cytoplasmic or membranous staining of IQGAP1 with an intensity of $1+$ (f), an example for IQGAP1 expression with a moderate intensity of $2+(\mathbf{g})$ or strong IQGAP1 staining with an intensity of $3+(\mathbf{h})$ are depicted. Adenocarcinoma negative for Tiam1 (i) or displaying weak membranous staining of Tiam1 with an intensity of $1+(\mathbf{j})$, an example for Tiam1 expression with a moderate intensity of $2+(\mathbf{k})$ or strong Tiam1 staining with an intensity of $3+(\mathbf{l})$ are shown. Gastric adenocarcinoma negative for E-cadherin (m) or displaying weak membranous staining of E-cadherin with an intensity of $1+(\mathbf{n})$, an example for E-cadherin expression with a moderate intensity of $2+(\mathbf{o})$ or strong E-cadherin staining with an intensity of $3+(\mathbf{p})$ are depicted. Original magnification $\times 400$. 
Table 2 Rac1, IQGAP1, Tiam1 and E-cadherin reactivity and its correlation with clinicopathologic features

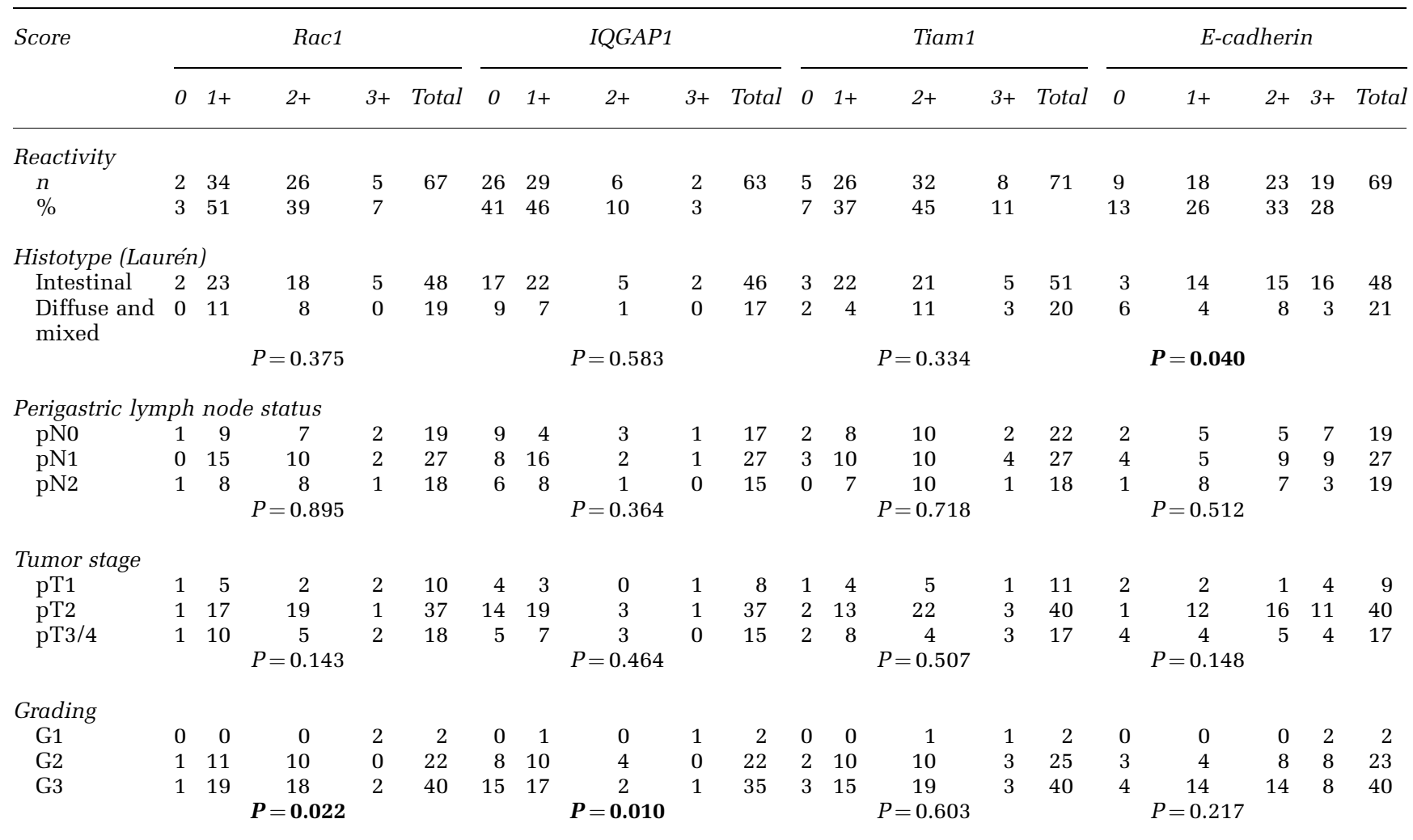

$P$-values were obtained by Pearson $\chi^{2}$ or Fisher's exact test; significant values are in bold.

in eight cases ( $11 \%$, score $3+$, Table 2). Expression of Tiam1 was found at the cellular membrane of tumor cells (Figure 1i-l).

E-cadherin reactivity was absent in nine cases ( $13 \%$, score 0$)$, weak in 18 cases $(26 \%$, score $1+)$, moderate in 23 cases (33\%, score $2+$ ) and strong in 19 cases $(28 \%$, score $3+$, Table 2). Expression of E-cadherin was detected at the cellular membrane of tumor cells (Figure $4 \mathrm{~m}-\mathrm{p}$ ).

\section{Correlation Analysis Between Expression Levels}

Correlation analysis between expression levels of Rac1, IQGAP1, Tiam1 and E-cadherin using the Spearman's rho test revealed a significant correlation between Rac1 and IQGAP1 expression $(P=0.007, r=0.343)$ and a trend suggesting an inverse correlation between Rac1 and E-cadherin expression $(P=0.055, r=-0.245)$ (Figure 2).

\section{Correlations with Clinical Data}

A significant correlation was observed between the presence of Rac1 and grading $(P=0.022)$ as well as between IQGAP1 and grading $(P=0.010$, Table 2). No association was observed between Tiam1 and pT, $\mathrm{pN}$, grading and classification (Table 2). E-cadherin staining was more prominent in intestinal-type

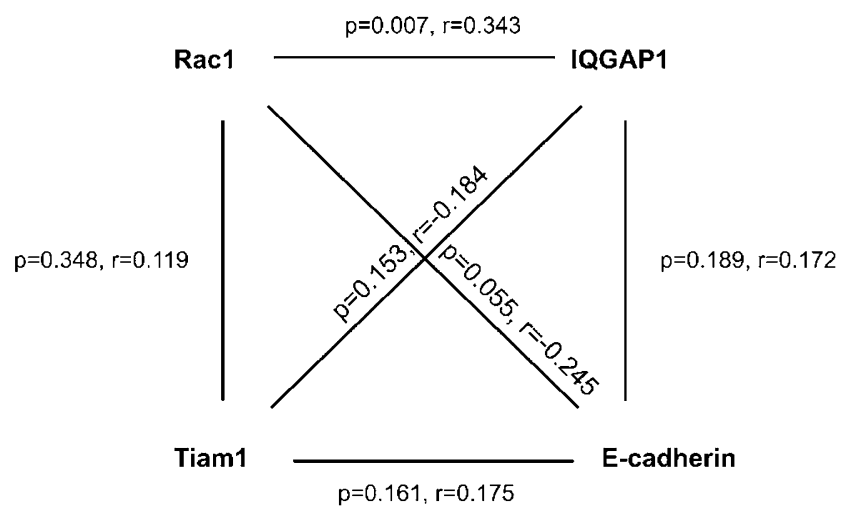

Figure 2 Correlation analysis. Correlation analysis was performed between the expression levels of Rac1, IAGAP1, Tiam1 and E-cadherin in gastric adenocarcinomas.

compared to diffuse- or mixed-type gastric cancer. The correlation between reactivity for E-cadherin and classification was significant $(P=0.040$, Table 2).

\section{Prognostic Significance of Rac1, IQGAP1, Tiam1 and E-cadherin}

Kaplan-Meier method was used to correlate the expression of Rac1, IQGAP1, Tiam1 and E-cadherin 
with patient survival. The mean and median of the overall patient follow-up were 57.9 or 38.8 months, respectively (range, 0.2-183.8 months, s.d. 51.7 months). No significant correlation with prognosis was observed for expression of Rac1 (Figure 3, log-rank 0.252). Trends were observed when the prognostic significance of IQGAP1 (Figure 4, logrank 0.088) or Tiam1 expression (Figure 5, log-rank $0.097)$ was investigated. No significant correlation between the expression of E-cadherin and prognosis was observed (Figure 6, log-rank 0.502).

\section{Expression of Rac1, IQGAP1 and Tiam1 in Gastric Cancer with E-cadherin Mutations}

Expression of Rac1, IQGAP1 and Tiam1 was also investigated in selected gastric cancer cases with E-cadherin mutations. The clinicopathological features of two patients with deletion of exon 9 of E-cadherin are shown in Table 3 . In patients with E-cadherin mutations, expression of Rac1, IQGAP1 and Tiam1 was low or absent (Table 4).

\section{Discussion}

We examined the expression in Rac1, the effector protein IQGAP1 and the activator Tiam1 in relation to E-cadherin in a series of 76 gastric adeno carcinomas in a tissue microarray study. Immunohistochemical investigation revealed increased expression (score $2+$ or $3+$ ) of Rac1 in $46 \%$ and of Tiam 1 in $56 \%$ of tumors. Expression (score 1-3+) of IQGAP1 was observed in 59\% and of E-cadherin in $87 \%$ of tumors. From our results, the following conclusions were drawn: (1) Rac1 and E-cadherin expression were not related to prognosis, while

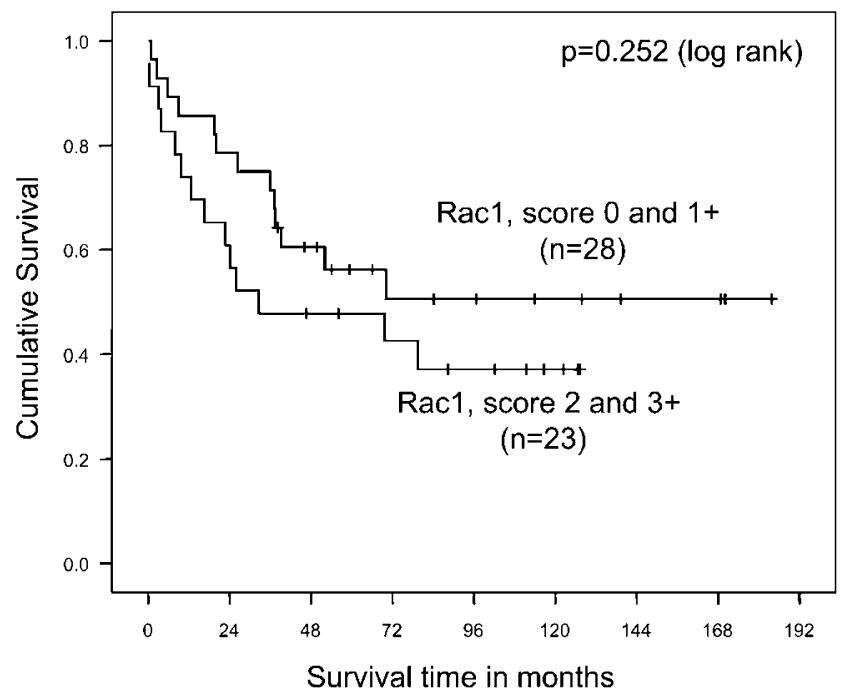

Figure 3 Survival impact of Rac1 expression. Kaplan-Meier survival curve for the gastric carcinoma patients stratified according to the Rac1 expression status in gastric carcinoma cells. The log-rank test statistical analysis indicates a $P=0.252$ (not significant). trends for a favorable prognosis of patients with Tiam1 expression and a lack of IQGAP1 expression were observed. (2) The expression levels of Rac1 and IQGAP1 were significantly correlated. (3) Tumors with E-cadherin mutations showed reduced levels or absence of Rac1, IQGAP1 and Tiam1.

\section{The Rho GTPase Rac1}

In this study, we detected increased expression of Rac1 in around half of the investigated gastric adenocarcinomas, a link between Rac1 expression and grading and a correlation between Rac1 and

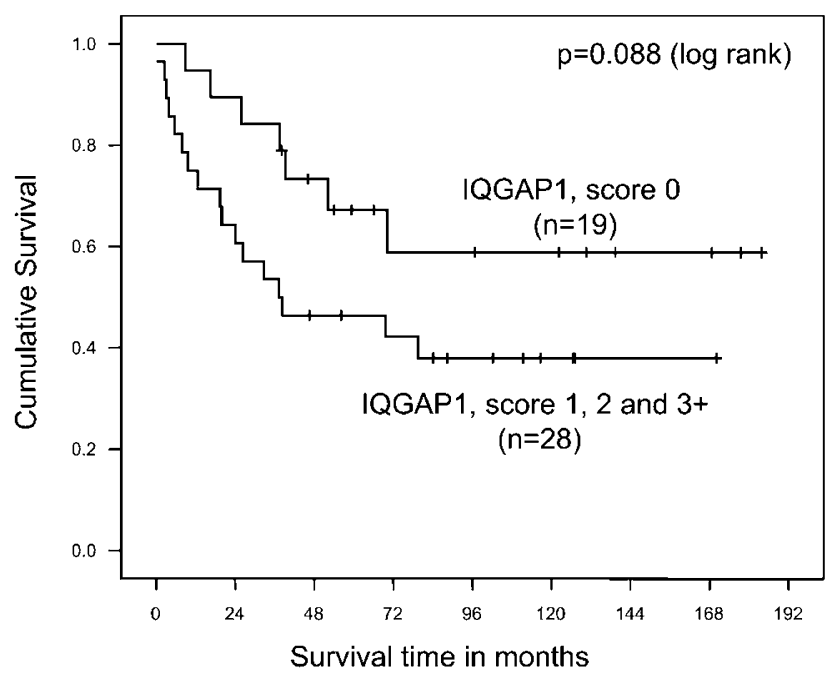

Figure 4 Survival impact of IQGAP1 expression. Kaplan-Meier survival curve for gastric carcinoma patients stratified according to the IQGAP1 expression status (score 0 vs score $1+, 2+$ and $3+)$ in gastric carcinoma cells. A cutoff of $10 \%$ was used. The log-rank test statistical analysis indicates a $P=0.088$ (trend).

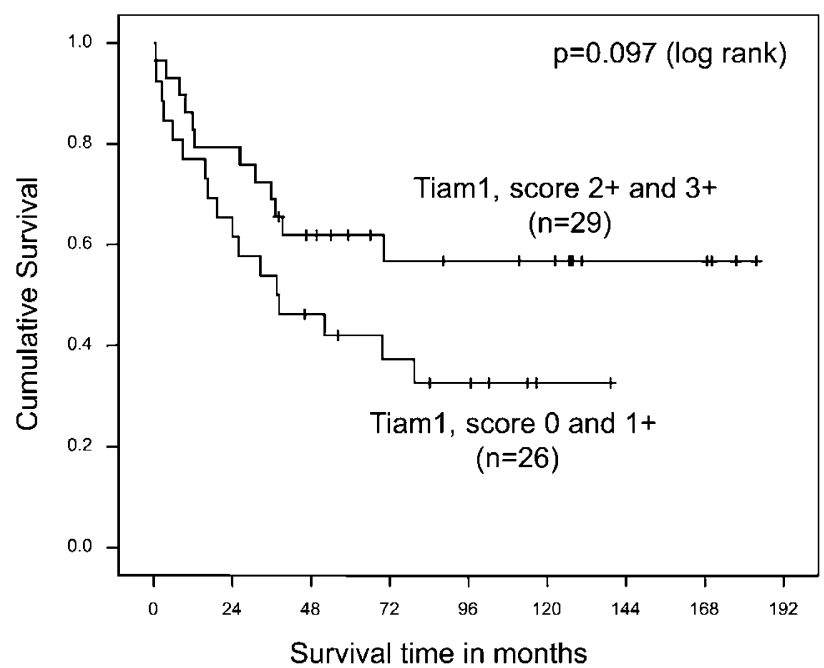

Figure 5 Survival impact of Tiam1 expression. Kaplan-Meier survival curve for gastric carcinoma patients stratified according to the Tiam 1 expression status (score 0 and $1+v s$ score $2+$ and $3+$ ) in gastric carcinoma cells. A cutoff of $10 \%$ was used. The log-rank test statistical analysis indicates a $P=0.097$ (trend). 


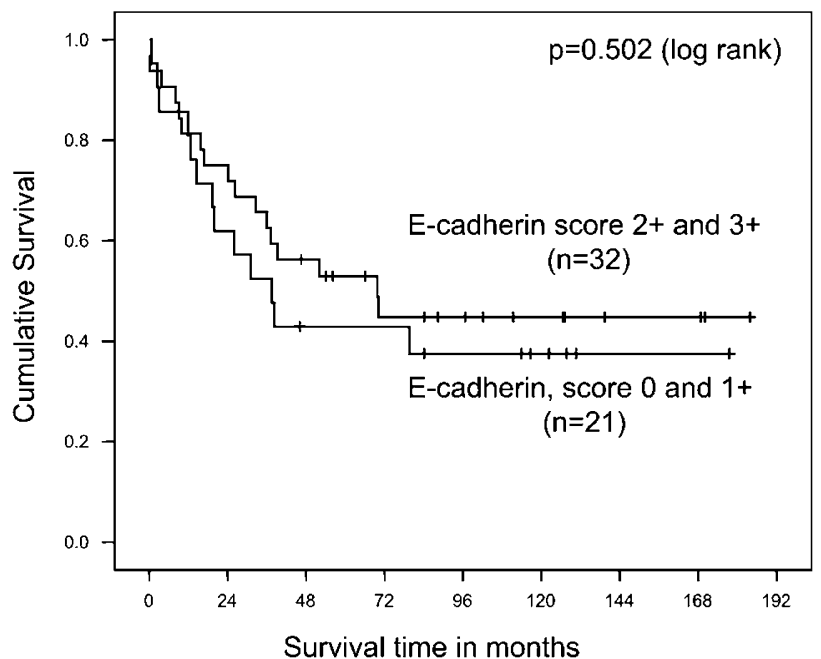

Figure 6 Survival impact of E-cadherin expression. KaplanMeier survival curve for gastric carcinoma patients stratified according to the E-cadherin expression status (score 0 and $1+v s$ score $2+$ or $3+$ ) in gastric carcinoma cells. The log-rank test statistical analysis indicates a $P=0.502$ (not significant)

Table 3 Clinicopathologic features of patients with diffuse-type gastric adenocarcinomas with del 9 E-cadherin mutations

\begin{tabular}{|c|c|c|}
\hline Patient & 1 & 2 \\
\hline $\begin{array}{l}\text { E-cadherin } \\
\text { mutation }\end{array}$ & del 9 & $\operatorname{del} 9$ \\
\hline Age & 73 & 42 \\
\hline Gender & Male & Female \\
\hline Histotype (Laurén) & $\begin{array}{l}\text { Diffuse-type signet ring } \\
\text { cell carcinoma }\end{array}$ & Diffuse-type \\
\hline $\mathrm{T}$ stage & pT2b & pT1 \\
\hline $\mathrm{N}$ stage & No & No \\
\hline G & 3 & 3 \\
\hline Residual disease & Ro & Ro \\
\hline Survival status & Dead & Alive \\
\hline $\begin{array}{l}\text { Survival time } \\
\text { (month) }\end{array}$ & 12.0 & - \\
\hline
\end{tabular}

IQGAP1 expression. Only few data are available in the literature that refers to the role of Rac1 in gastric cancer. Recent findings indicate that Rac1 may play an important role in the carcinogenesis and progression of gastric carcinoma, because increased Rac1 expression was related to higher TNM stages. ${ }^{22}$ Genetic deletion of Rac1 has revealed its critical role in cell survival regulation ${ }^{23}$ suggesting that increased expression of Rac1 in tumors may protect cells from apoptosis and lead to an increase of cellmatrix-interaction and cell spread. However, despite these important functions that are attributed to Rac1, no association of expression of Rac1 with prognosis was found in our study of gastric carcinomas. Of note, the investigated cohort was of relatively small size, limiting the conclusions that can be drawn.
Table 4 Expression of Rac1, Tiam1 and IQGAP1 in diffuse-type gastric adenocarcinomas with del 9 E-cadherin mutations

\begin{tabular}{lll}
\hline Patient & 1 & 2 \\
\hline E-cadherin mutation & del 9 & del 9 \\
E-cadherin & $3+$ & $3+$ \\
Rac1 & 0 & $1+$ \\
IQGAP1 & $1+$ & $1+$ \\
Tiam1 & $1+$ & $1+$ \\
\hline
\end{tabular}

\section{The Rac1 Downstream Effector IQGAP1}

We provide evidence that IQGAP1, a downstream effector of Rac1, is expressed in more than half of the examined gastric carcinomas and that its expression is linked to grading. Further, we demonstrate a trend that a lack of expression of IQGAP1 is beneficial for the patient's survival. These results are consistent with in vitro data, showing that IQGAP1 negatively regulates E-cadherin-mediated cell-cell adhesion by interacting with $\beta$-catenin and displacing $\alpha$-catenin from the adherens complex. In its activated GTPbound form, Rac1 sequesters IQGAP1 and prevents its binding to $\beta$-catenin, thereby stabilizing cadherin-mediated cell adhesion. ${ }^{24}$ The role of IQGAP1 in gastric carcinogenesis has been studied by several other groups, but to our knowledge, data on the prognostic significance of IQGAP1 in gastric cancer has not been reported. An inverse correlation of IQGAP1 with intercellular adhesion was shown in gastric cancers with cytoplasmic localization of IQGAP1 in intestinal type gastric cancer and membranous localization of IQGAP1 at the cellular membrane in diffuse type gastric cancer cells without cell-cell contact. ${ }^{25}$ IQGAP1 expression has also been studied in other types of tumors. In colorectal cancer, overexpression of IQGAP1 in carcinomas and its association with invasion fronts has been detected. ${ }^{26}$ In ovarian cancer, overexpression and diffuse expression pattern of IQGAP1 at invasion fronts are independent prognostic parameters. ${ }^{27}$ To conclude, these reports and our own results converge at a negative role for IQGAP1 in tumorigenesis.

\section{The Rac1-activator Tiam1}

Here we report increased expression of the Rac1activator Tiam1 in half of the examined gastric carcinomas. Further, increased positivity of tumor cells for Tiam1 tended to be associated with favorable prognosis. Tiam1 is required for the establishment and maintenance of E-cadherin-based adhesions, ${ }^{28}$ hence it seems to play a role in conserving the epithelial phenotype of cells. In line with this, Tiam1-mediated activation of Rac1 stimulates cadherin-mediated cell adhesion, thereby limiting the capacity of cells to migrate and to 
invade. ${ }^{6}$ On the other side, the function of Tiam1 strongly depends on the cellular context, because Tiam1 has originally been identified as an invasionand metastasis-inducing gene in a murine T-lymphoma cell line. ${ }^{29}$ Concerning cancer onset, Tiam1 has been found to correlate both positively and negatively with tumor progression, depending on the type of cancer. ${ }^{3}$ Tiam1 expression correlated inversely with renal tumor invasion in vitro and mutations in Tiam1 have been found in renal cell carcinoma. ${ }^{30}$ However, Tiam1 plays an important role in the progression and metastasis of cancers, including colon and breast carcinomas. ${ }^{31}$

\section{E-cadherin}

In this study, we found expression of E-cadherin in the majority of cases. In accordance with previous results, the protein level has low impact on patient survival. ${ }^{21}$ Here we show that there was a tendency towards an inverse correlation between expression of E-cadherin and Rac1. Mutation of E-cadherin is frequently observed in diffuse-type gastric carcinoma. ${ }^{17}$ We recently demonstrated that patients carrying deletions of exons 8 or 9 , observed in $5 \%$ of tumors in a Mexican series of gastric carcinomas, had a worse prognosis than patients without these alterations. ${ }^{21}$ Here we report that expression of Rac1, IQGAP1 and Tiam1 was low or absent in tumor cases with E-cadherin mutations. This result indicates that the trend for the inverse correlation between expression of E-cadherin and Rac1 is preserved in the presence of the mutation.

Taken together, in this study we investigated whether expression of Rac1, IQGAP1 and Tiam1 is deregulated in gastric cancer. We present evidence that upregulation (for Rac1 and IQGAP1) as well as downregulation (for Tiam1 and E-cadherin) occurs. Expression of Rac1 was positively linked to IQGAP1 and tended to be inversely linked to E-cadherin. Further studies with larger numbers of tumors are necessary to finally establish the role of the investigated markers in gastric carcinogenesis.

\section{Acknowledgements}

We thank C Hartmann, M Beckesch and A Voss for excellent technical assistance. Our study was supported by a grant to B Luber from the Deutsche Krebshilfe (Nr 106148).

\section{References}

1 Cavallaro U, Christofori G. Cell adhesion and signalling by cadherins and Ig-CAMs in cancer. Nat Rev Cancer 2004;4:118-132.

2 Bar-Sagi D, Hall A. Ras and Rho GTPases: a family reunion. Cell 2000;103:227-238.
3 Gomez del Pulgar T, Benitah SA, Valeron PF, et al. Rho GTPase expression in tumourigenesis: evidence for a significant link. Bioessays 2005;27:602-613.

4 Braga VM, Machesky LM, Hall A, et al. The small GTPases Rho and Rac are required for the establishment of cadherin-dependent cell-cell contacts. J Cell Biol 1997;137:1421-1431.

5 Michiels F, Habets GG, Stam JC, et al. A role for Rac in Tiam1-induced membrane ruffling and invasion. Nature 1995;375:338-340.

6 Hordijk PL, ten Klooster JP, van der Kammen RA, et al. Inhibition of invasion of epithelial cells by Tiam1-Rac signaling. Science 1997;278:1464-1466.

7 Kuroda S, Fukata M, Kobayashi K, et al. Identification of IQGAP as a putative target for the small GTPases, Cdc42 and Rac1. J Biol Chem 1996;271:23363-23367.

8 Hart MJ, Callow MG, Souza B, et al. IQGAP1, a calmodulin-binding protein with a rasGAP-related domain, is a potential effector for cdc42Hs. EMBO J 1996;15:2997-3005.

9 Kuroda S, Fukata M, Nakagawa M, et al. Role of IQGAP1, a target of the small GTPases Cdc42 and Rac1, in regulation of E-cadherin-mediated cell-cell adhesion. Science 1998;281:832-835.

10 Tahara E. Molecular aspects of invasion and metastasis of stomach cancer. Verh Dtsch Ges Pathol 2000;84: 43-49.

11 Becker KF, Atkinson MJ, Reich U, et al. E-cadherin gene mutations provide clues to diffuse type gastric carcinomas. Cancer Res 1994;54:3845-3852.

12 Muta H, Noguchi M, Kanai Y, et al. E-cadherin gene mutations in signet ring cell carcinoma of the stomach. Jpn J Cancer Res 1996;87:843-848.

13 Tamura G, Sakata K, Nishizuka S, et al. Inactivation of the E-cadherin gene in primary gastric carcinomas and gastric carcinoma cell lines. Jpn J Cancer Res 1996;87: 1153-1159.

14 Machado JC, Soares P, Carneiro F, et al. E-cadherin gene mutations provide a genetic basis for the phenotypic divergence of mixed gastric carcinomas. Lab Invest 1999;79:459-465.

15 Oda T, Kanai Y, Oyama T, et al. E-cadherin gene mutations in human gastric carcinoma cell lines. Proc Natl Acad Sci USA 1994;91:1858-1862.

16 Becker KF, Atkinson MJ, Reich U, et al. Exon skipping in the E-cadherin gene transcript in metastatic human gastric carcinomas. Hum Mol Genet 1993;2:803-804.

17 Berx G, Becker KF, Hofler H, et al. Mutations of the human E-cadherin (CDH1) gene. Hum Mutat 1998;12: 226-237.

18 Hamilton S, Aaltonen LA (eds). World Health Organization classification of tumours. Pathology and Genetics of Tumours of the Digestive System. IARC Press: Lyon, 2000.

19 Sobin L, Wittekind Ch (eds). UICC: TNM Classification of Malignant Tumours, 6th edn. Wiley-Liss: New York, 2002.

20 Becker KF, Kremmer E, Eulitz M, et al. Analysis of E-cadherin in diffuse-type gastric cancer using a mutation-specific monoclonal antibody. Am J Pathol 1999;155:1803-1809.

21 Gamboa-Dominguez A, Dominguez-Fonseca C, Chavarri-Guerra Y, et al. E-cadherin expression in sporadic gastric cancer from Mexico: exon 8 and 9 deletions are infrequent events associated with poor survival. Hum Pathol 2005;36:29-35. 
22 Pan Y, Bi F, Liu N, et al. Expression of seven main Rho family members in gastric carcinoma. Biochem Biophys Res Commun 2004;315:686-691.

23 Guo F, Debidda M, Yang L, et al. Genetic deletion of Rac1 GTPase reveals its critical role in actin stress fiber formation and focal adhesion complex assembly. J Biol Chem 2006;281:18652-18659.

24 Fukata M, Kuroda S, Nakagawa M, et al. Cdc42 and Rac1 regulate the interaction of IQGAP1 with betacatenin. J Biol Chem 1999;274:26044-26050.

25 Takemoto H, Doki Y, Shiozaki H, et al. Localization of IQGAP1 is inversely correlated with intercellular adhesion mediated by e-cadherin in gastric cancers. Int J Cancer 2001;91:783-788.

26 Nabeshima K, Shimao Y, Inoue T, et al. Immunohistochemical analysis of IQGAP1 expression in human colorectal carcinomas: its overexpression in carcinomas and association with invasion fronts. Cancer Lett 2002;176:101-109.
27 Dong P, Nabeshima K, Nishimura N, et al. Overexpression and diffuse expression pattern of IQGAP1 at invasion fronts are independent prognostic parameters in ovarian carcinomas. Cancer Lett 2006;243:120-127.

28 Malliri A, van Es S, Huveneers S, et al. The Rac exchange factor Tiam 1 is required for the establishment and maintenance of cadherin-based adhesions. J Biol Chem 2004;279:30092-30098.

29 Habets GG, Scholtes EH, Zuydgeest D, et al. Identification of an invasion-inducing gene, Tiam-1, that encodes a protein with homology to GDP-GTP exchangers for Rho-like proteins. Cell 1994;77:537-549.

30 Engers R, Zwaka TP, Gohr L, et al. Tiam1 mutations in human renal-cell carcinomas. Int J Cancer 2000;88: 369-376.

31 Minard ME, Kim LS, Price JE, et al. The role of the guanine nucleotide exchange factor Tiam1 in cellular migration, invasion, adhesion and tumor progression. Breast Cancer Res Treat 2004;84:21-32. 